\title{
A Review Paper on Attendance Marking System based on Face Recognition
}

\author{
Khem Puthea, Rudy Hartanto and Risanuri Hidayat
}

\begin{abstract}
Attendance marking system has been become a challenging, intriguing and accurately in the real-time system. It is tough to mark the attendance of a student in the large classroom, and there are many students attend the class. Many attendance management systems have been implemented in the current research. However, the attendance management system by using facial recognition still has issues which allow the research to improve the current research to make the attendance management system working well. The paper will do a literature review on the previous worked from different researcher has done on their research paper. This paper does not only provide the literature review on the earlier work or related work, but it also provide the deep analysis of Principal Component Analysis, discussion, suggestion for future work.
\end{abstract}

Keywords-PCA, Face Recognition, Histogram Oriented Gradient, Automatic System, AMS.

\section{INTRODUCTION}

$\mathrm{F}$ ACE Recognition has received many interests in recent years of face recognition development and has become a popular research. Moreover, it is a critical application in image analysis, and it is a very challenge to create an automated system based on face recognition; which has an ability to recognize human face accuracy. Solving the manual attendance problem and time-consuming, many research has been conducted with the automated [1], [2], [3] or smart attendance management [4] system to resolve the issues of manual attendance. However, the biometric attendance management system is tough to verify each student in the classroom which there are many students attend the class, and it interrupts the student when the system response to the student if the system cannot support detection or recognition. Also, the biometric system needs more hardware or interaction with the student who needs to spend some budget on the accessories and sometimes still has time-consuming. The research on real-time face recognition has been a real challenging to build with attendance management system. Automatic attendance marking can solve the central issues such as the error in the manual attendance when the academic office input the data from the sheet into the system. As we know that we cannot make sure the data in the university office is correct $100 \%$, this is a concerned when we use the

Khem Puthea, Universitas Gajah Mada Yogyakarta, Indonesia (corresponding author's phone:; fax:; ).

Rudy Hartanto, Universitas Gajah MadaYogyakarta, Indonesia

Risanuri Hidayat, Universitas Gajah Mada Yogyakarta, Indonesia traditional method of the attendance marking system in the university which as many students enrolled in each year, has many departments, and many class in the college. The face recognition process has many steps such as capture, extraction, comparison, matching [5]. The operate in each process are:

Step one: capture is the way to snap the picture during the enrollment of the system. Then second step, extraction is used for finding or extract the specific object from the face. Then the comparison step, new input are used for comparing with the enrollment or the database (sample data). The last step is matching: the system will be possible to find the matching of the new face with the registration face or not based on extraction and comparison process.

From the following above we can illustrate as Figure 1 below

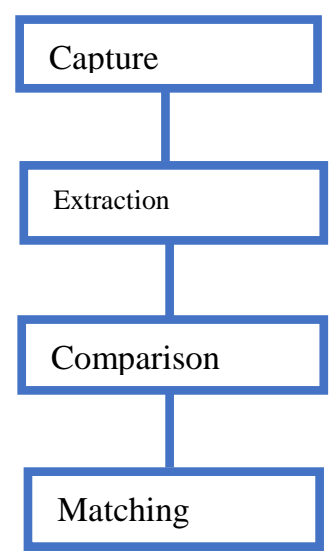

Fig 1. The operate process of face recognition system

According to the above process, facial recognition is not an easy task to do. There are several methods and algorithm needed to implement with face recognition. In [6] has implemented the system to recognize the human face with LBP( Local Binary Pattern) and using SVM(Support Vector Machine) to find the similarities of the images stored in the database with the acquired image snap by the camera inside the classroom.

\section{FACE RECOGNITION}

In the introduction section already given an overview of face recognition in attendance marking system. In part will discuss the various approaches to face recognition such as:

\section{A. Geometric Approach:}

This approach is recognized human-based on geometry 
point on the face. There are many features of geometric approach has been conducted : [7] used geometric approach to improve the accuracy of the system in case the images contains the complex background.

\section{B. PCA Based:}

PCA is a statistical method has been used in face recognition for feature extraction and used to remove the redundancy information. PCA also used to decrease dimensionality, and PCA also used to conserve the information. Given images into the system; face recognition will process to find the matching image between the given images with the images inside the databases. Principal component or Eigenface is a face that contains feature and characteristic. The image with the can be written to 1dimensional to reduce the dimensionality.

\section{C.LDA-Based:}

Is used to maximize the scatter image in the same class or in a different class which is enhanced from PCA. In PCA is selected subspace that contains most of the variation. In 1997 "Fisherfaces" [8] has been used in the application of (FLD) Fisher's linear discriminant. The Fisher's linear discriminant (FLD) choose the linear subspace $\Phi$ that makes the ration more maximize.

$$
\frac{\left|\Phi^{T} S_{b} \Phi\right|}{\left|\Phi^{T} S_{w} \Phi\right|}
$$

Where

$$
S_{b}=\sum_{i=1}^{m} N_{i}\left(\bar{x}_{i}-\bar{x}\right)\left(\bar{x}_{i}-\bar{x}\right)^{T}
$$

moreover, $S_{w}=\sum_{i=1}^{m} \sum_{x \in X_{i}}\left(x-\bar{x}_{i}\right)\left(x-\bar{x}_{i}\right)^{T}$

$S_{b}$ between-class scatter

$S_{w}$ within-class scatter

$m$ number of subject in the database

In [8] author was implemented and tested with a frontal face, and the result contains error less than an implementation with Eigenfaces.

\section{D.LBP(Local Binary Pattern)}

Is an approach used in computer vision, first introduced in 1990 used for classification. In 1994 based on research papers [9] and [10] shown that LBP implements with HOG(Histogram of Oriented Gradient) having a great power feature for texture classification and improve the performance detection on some relevant data sets.

\section{E. Active Appearance Model:}

Was introduced first by Edwards, Cootes and Taylor [11], [12] in the same year. This approach has been used in the extensive area to do matching the face and using in the field of medical interpretation.

\section{FACE RECOGNITION TECHNIQUES}

\section{A. Principal Component Analysis( PCA)}

PCA knew as dimension reduction and feature selection, however, PCA used to extract the number of principal component of the data has multidimensional. Human Recognition system using Principal Component Analysis was developed firstly by Turk and Pentland [13] which was solve face recognition in two-dimensional rather than threedimensional geometry. Because both of the captured image and basic vector constructed by the PCA has the same dimension [14] and reconstruction the human face has been done by [15] used for solving the retrieval large database images. In the Recent development of automatic face recognition, the feature extraction has become significant to extract features such as nose, eyes, and mouth. Moreover, the main goal of PCA also keeps the original information of the data, to minimize original loss information and to improve the face analysis. The Principal Component Analysis is the mainly of Eigenfaces was extremely large confined to dimension reduction [16]. In [17] has been introduced a way to do calculating the discriminant feature of PCA by using a recursive algorithm. Now we are focus on the step of PCA in face recognition system by using Eigenface

- Capture and prepare image for training

Suppose we have images for training in face recognition system. The given images must be the same size.

- All the face in is transformed as a vector and put into a training set.

$$
S=\left\{\Gamma_{i}, \ldots, \Gamma_{n}\right\}
$$

Which we assume images has the size $N \times N$ or $N^{2}$ dimensional.

- Compute the mean face

$$
\bar{X}=\frac{1}{N} \sum_{i=1}^{i=n} x_{n}
$$

- Compute the average of face vector

The mean face subtracts by original face and the result will have stored in variable

$$
\Phi_{i}=\Gamma_{i}-\bar{X}
$$

- Compute the covariance

Calculate the covariance matrix with the following mathematic formula in the following

$$
C=S=\frac{\sum_{i=1}^{n}\left(\Gamma_{i}-\bar{X}\right)\left(\Gamma_{i}-\bar{X}\right)^{T}}{n}
$$

- Calculate the covariance matrix of Eigenvector and Eigenvalues

The new face is transformed into its eigenface components and the resulting weights from the weight vectors.

Where $\omega$ = weight, $\mu$ = eigenvector, $\Gamma=$ new input image, $\bar{X}=$ mean face 
The weight vector $\Omega^{T}$ is given by,

$$
\Omega^{T}=\left\{\omega_{1}, \omega_{2}, \ldots, \omega_{n}\right\}
$$

Then Eigenfaces handle face recognition:

-Training data project into PCA subspace

-Query image project into PCA subspace

-Find the nearest neighbor between the query image and training image which already projects into PCA subspace.

\section{LITERATURE SURVEY}

The primary purpose of this paper review is to find the solutions provided by others author and consider the imperfection of the system proposed by them, give the best solutions.

In [18] Kawaguchi introduced a lecture attendance system with a new method called continuous monitoring, and the student's attendance marked automatically by the camera which captures the photo of a student in the class. The architecture of the system is simple since two cameras equipped with the wall of the class. The first one is a capturing camera used to capture the image student in the class and the second camera is sensor camera is used to getting the seat of a student inside the class and the camera capturing will snap the image of the student. The system compares the picture taking from a camera capturing images and faces in the database done much time to perfect the attendance.

Other paper proposed by [2] introduced a real-time computer vision algorithm in automatic attendance management system. The system installed the camera with non-intrusive, which can snap images in the classroom and compared the extracted face from the image of the camera capturing with faces inside the system. This system also used machine learning algorithm which are usually used in computer vision. Also, HAAR CLASSIFIERS used to train the images from the camera capturing. The face snap by the camera capturing will convert to grayscale and do subtraction on the images; then the image is transferred to store on the server and processing later.

In 2012 N. Kar [19] introduced an automated attendance management system using face recognition technique which used the Principal Component Analysis To implementation the system, use two libraries such OpenCV is a computer vision library and FLTK(Light Tool Kit. Both of this libraries helped the development such as OpenCV support algorithm [20] and FLTK [21] used to design the interface. In the system, there are Request Matching and Adding New fact to Database. In Request Matching, the first step is open the camera and snap the photo after the extraction the frontal face. The next step is recognizing the face with the training data and project the extracted face onto the Principal Component Analysis. The final step displays the nearest face with the acquired images. Apart from that, adding a new face into the database is snap the photo after that extract the frontal face images and then perform the Haar cascade Method to find the object in the image in different window size. The next step is to store the image into the database and to learn the face then perform the Principal Component Analysis Algorithm. The final step is storing the information inside the face XML file. The system is focused on the algorithm to improve the face detection from acquired images or videos.

In [3] the author also proposed a system which implements automatic attendance using face recognition. The system which can extract the object in the face such nose, mouth by using MATLAB with Principal Component Analysis (PCA). The system [7] designed to resolve the issues of attendance marking system such as time-consuming. As the result of the experiment show that this paper, the system can recognize in case the dark background or difference view of the face in the classroom.

Jyotshana Kanti [4] proposed a smart attendance marking system which combines two differencing algorithms such Principal Component Analysis and Artificial Neural Network. The purpose of the author is to solve the traditional attendance marking system and to resolve the time-consuming. In the system implement with Principal Component Analysis, it does an extraction and identify the similarities of the face database and acquire images. Artificial Neural Network is used to solve the problem of the input data or learn from the input data, and the expect value. In the system implemented by the author using back propagation algorithm and combines with mathematical function to perform in that system. As a result, written by the author research, it shows that the system can use to recognize in a different environment.

In [22] Priyanka Thakare proposed a method using Eigenface and Principal Component Analysis which has the architecture as the following step. The camera needs to install in the front which can capture an entire face of the student inside the class. The first phase after the camera has been captured; the captured image was transferred into the system as an input. The image capture from the camera sometimes come with the darkness or brightness which need to do an enhancement on it such as convert to gray image. The next step, Histogram Normalization is used in this system remove the contrast of the image. It is easy to recognize when has the student sit in the back row. The Median filter is used to remove noise from the image in case the camera is high definition camera, but sometimes it still contains the noise. The author also implements with skin classification which changes all the pixel to black except the pixel are close to the skin.

\section{COMPARISON Of FACE RECOGNITION TECHNIQUES}

Various technical papers have shown the studies of number each algorithm. This table covers five papers that are related to this area, and an overview of all the papers survey is given here we give mainly their problems and limitations we also give a summary and conclusion every researcher has proposed in those papers. 
TABLE I

SUMMARY PAPERS

\begin{tabular}{|c|c|c|c|}
\hline Author & Algorithm & Problem & Summary \\
\hline Visar Shehu & PCA & $\begin{array}{c}\text { The recognition } \\
\text { rate is } 56 \%, \\
\text { having a problem } \\
\text { to recognize } \\
\text { student in year } 3 \text { or } \\
4\end{array}$ & $\begin{array}{l}\text { Using HAAR } \\
\text { Classifier and } \\
\text { computer vision } \\
\text { algorithm to } \\
\text { implement face } \\
\text { recognition }\end{array}$ \\
\hline Syen navaz & $\begin{array}{l}\text { PCA, } \\
\text { ANN }\end{array}$ & $\begin{array}{l}\text { Low accuracy with } \\
\text { the big size of } \\
\text { images to train } \\
\text { with PCA }\end{array}$ & $\begin{array}{l}\text { Using PCA to train } \\
\text { and reduce } \\
\text { dimensionality and } \\
\text { ANN to classify input } \\
\text { data and find the } \\
\text { pattern }\end{array}$ \\
\hline $\begin{array}{c}\text { Kar, } \\
\text { Nirmalya }\end{array}$ & PCA & $\begin{array}{l}\text { Repeat image } \\
\text { capturing }\end{array}$ & $\begin{array}{l}\text { Using Eigenvector } \\
\text { and Eigenvalue for } \\
\text { face recognition }\end{array}$ \\
\hline $\begin{array}{l}\text { Joseph, } \\
\text { Jomon }\end{array}$ & $\begin{array}{l}\text { PCA and } \\
\text { Eigenfaces }\end{array}$ & $\begin{array}{l}\text { Validation of the } \\
\text { student once } \\
\text { marked present is } \\
\text { not done }\end{array}$ & $\begin{array}{l}\text { Using PCA with } \\
\text { MATLAB to } \\
\text { implement face } \\
\text { recognition }\end{array}$ \\
\hline Nirmalya & $\begin{array}{l}\text { PCA, } \\
\text { ANN }\end{array}$ & $\begin{array}{c}\text { Hight } \\
\text { Computational } \\
\text { cost due to } \\
\text { combining PCA } \\
\text { and ANN }\end{array}$ & $\begin{array}{c}\text { Using PCA and ANN } \\
\text { to do a better } \\
\text { attendance result }\end{array}$ \\
\hline Neerja & PCA & $\begin{array}{l}\text { Low accuracy in } \\
\text { lighting }\end{array}$ & $\begin{array}{l}\text { Combine PCA with } \\
\text { fuzzy feature } \\
\text { extraction and dataset } \\
\text { tested with Indian } \\
\text { people }\end{array}$ \\
\hline
\end{tabular}

In the artificial intelligence-based method, Artificial Neural Network and has proved the result of the experiment indicating that Artificial Neural Network is shown itself to be successful in classification and system performance compared to some other artificial intelligence-based algorithm or conventional algorithm. On the top of that, the integration of the traditional and artificial intelligence-based was presented in previous paper namely "Face recognition using Principal Component analysis and Neural Network" has been achieved in term of accuracy and performance to previous attendance management based on face recognition. The various paper has been implemented with Principal Component Analysis with AdaBoost [23], PCA with Error Back-Propagation [24] [25], PCA with ANN and Wavelet [26]. Each paper show that the accuracy of the system is higher than the previous worked which PCA have not incorporated with ANN.

\section{CONCLUSION}

After analyzing various methods, this paper can achieve the goal of analyzing each method such as overall system capacity, throughput as well as accuracy. According to analysis on those paper show that the PCA algorithm has been proved to be incredibly effective in an extensive database. After analysis to each paper, PCA is better in the System of Attendance Management Based on Face Recognition, is a technique used for securing the attendance, replacing the manual attendance system which is time-consuming and Convolutional Neural Network also contribute in attendance management system using face recognition due to the strong classifier. In future work, the accuracy of the system should be solved by incorporate principal component analysis with the convolutional neural network. The next research intends to get good generalization abilities. In [27] has been integrated ANN with PCA to solved a blocking issued of attendance management system using face recognition. However, it still has issues with performance and the accuracy of the system to recognize a human face. In the next research work will conduct fast PCA with back-pro Back-Propagation to resolved that problem.

\section{REFERENCES}

[1] A. Ahmedi and S. Nandyal, "An Automatic Attendance System Using Image processing," pp. 1-8, 2015.

[2] V. Shehu and A. Dika, "Using real time computer vision algorithms in automatic attendance management systems," Inf. Technol. Interfaces (ITI), 2010 32nd Int. Conf., pp. 397-402, 2010.

[3] J. Joseph and K. P. Zacharia, "Automatic Attendance Management System Using Face Recognition,” Int. J. Sci. Res., vol. 2, no. 11, pp. 327-330, 2013

[4] J. Kanti and A. Papola, "Smart Attendance using Face Recognition with Percentage Analyzer," vol. 3, no. 6, pp. 7321-7324, 2014.

[5] T. H. Le, "Applying Artificial Neural Networks for Face Recognition," Adv. Artif. Neural Syst., 2011.

[6] P. Mehta, "An Efficient Attendance Management Sytem based on Face Recognition using Matlab and Raspberry Pi 2," Int. J. Eng. Technol. Sci. Res. IJETSR, vol. 3, no. 5, pp. 71-78, 2016.

[7] S. Jeng, H. Y. M. Liao, C. C. Han, M. Y. Chern, and Y. T. Liu, "Facial feature detection using geometrical face model: An efficient approach," Pattern Recognit., vol. 31, no. 3, pp. 273-282, 1998.

[8] P. N. Belhumeur, J. P. Hespanha, and D. J. Kriegman, "Eigenfaces vs. fisherfaces: Recognition using class specific linear projection," IEEE Trans. Pattern Anal. Mach. Intell., 1997.

[9] T. Ojala, M. Pietikainen, and D. Harwood, "Performance evaluation of texture measures with classification based on Kullback discrimination of distributions," in Pattern Recognition, 1994. Vol. 1 - Conference A: Computer Vision amp; Image Processing., Proceedings of the 12th IAPR International Conference on, 1994.

[10] T. Ojala, M. Pietikäinen, and D. Harwood, "A comparative study of texture measures with classification based on feature distributions," Pattern Recognit., 1996.

[11] G. J. Edwards, C. J. Taylor, and T. F. Cootes, "Interpreting face images using active appearance models," Proc. Third IEEE Int. Conf. Autom. Face Gesture Recognit., 1998.

[12] T. F. Cootes, G. J. Edwards, and C. J. Taylor, Active appearance models. 1998.

[13] M. Turk and A. Pentland, "Eigenfaces for Recognition," J. Cogn. Neurosci., vol. 3, no. 1, pp. 71-86, 1991.

[14] W. Haider, H. Bashir, A. Sharif, I. Sharif, and A. Wahab, "A Survey on Face Detection and Recognition Techniques," Res. J. Recent Sci., 2014.

[15] D. L. Swets, "Using discriminant eigenfeatures for image retrieval," IEEE Trans. Pattern Anal. Mach. Intell., vol. 18, no. 8, pp. 831-836, 1996.

[16] L. C. Paul and A. Al Sumam, "Face Recognition Using Principal Component Analysis Method," vol. 1, no. 2012, pp. 135-139, 2012.

[17] I. Dagher, "Incremental PCA-LDA algorithm," in CIMSA 2010 - IEEE International Conference on Computational Intelligence for Measurement Systems and Applications, Proceedings, 2010.

[18] Y. Kawaguchi, "Face Recognition-based Lecture Attendance System," 3rd AEARU ..., no. October, 2005.

[19] N. Kar, M. K. Debbarma, A. Saha, and D. R. Pal, "Study of Implementing Automated Attendance System Using Face Recognition Technique," Int. J. Comput. Commun. Eng., vol. 1, no. 2, pp. 100-103, 2012. 
[20] "About - OpenCV library." [Online]. Available: http://opencv.org/about.html. [Accessed: 19-Apr-2017].

[21] "Fast Light Toolkit - Fast Light Toolkit (FLTK)." [Online]. Available: http://www.fltk.org/index.php. [Accessed: 19-Apr-2017].

[22] P. Wagh, R. Thakare, J. Chaudhari, and S. Patil, "Attendance system based on face recognition using eigen face and PCA algorithms," in 2015 International Conference on Green Computing and Internet of Things (ICGCIoT), 2015, pp. 303-308.

[23] K. Susheel Kumar, S. Prasad, V. Bhaskar Semwal, and R. C. Tripathi, "Real Time Face Recognition Using AdaBoost Improved Fast PCA Algorithm," Int. J. Artif. Intell. Appl., vol. 2, no. 3, pp. 45-58, Jul. 2011.

[24] J.-M. Kim and M.-A. Kang, "A Study of Face Recognition Using the PCA and Error Back-Propagation," in 2010 Second International Conference on Intelligent Human-Machine Systems and Cybernetics, 2010, vol. 2, no. 1, pp. 241-244.

[25] R. Toufiq and M. R. Islam, "Face recognition system using PCA-ANN technique with feature fusion method," Electr. Eng. Inf. Commun. Technol. (ICEEICT), 2014 Int. Conf., pp. 1-5, 2014.

[26] M. Mazloom and S. Ayat, "Combinational Method for Face Recognition: Wavelet, PCA and ANN," in 2008 Digital Image Computing: Techniques and Applications, 2008, pp. 90-95.

[27] A. S. S. NAVAZ and T. D. S. P. MAZUMDER, "Face Recognition using Principal Component Analysis and Neural Networks," vol. 1, no. April, pp. 91-94, 2001. 